\title{
Fairness Enhancement of Link Adaptation Techniques in Wireless Access Networks
}

\author{
Mohamed H. Ahmed \\ Dept. of Electrical \& Computer Engineering \\ Memorial University of Newfoundland \\ St. John's, Canada \\ mhahmed@engr.mun.ca
}

\author{
Halim Yanikomeroglu and Samy Mahmoud \\ Broadband Commun. \& Wireless Systems (BCWS) Centre \\ Dept. of Systems \& Computer Engineering \\ Carleton University, Ottawa, Canada \\ \{halim,mahmoud\}@sce.carleton.ca
}

\begin{abstract}
Link adaptation techniques, such as power control and adaptive coding and modulation, aim at maximizing the throughput in wireless networks while maintaining the signal quality in terms of the signal to interference ratio. However, fair resource allocation among different users must be taken into consideration, particularly in fixed broadband wireless access networks. The low/no mobility of users in such networks can lead to location-dependent resource utilization, which causes a significant variation in the performance from a user to another. For instance, adaptive coding and modulation schemes increase the aggregate throughput in the network; however, they also increase the variation of the throughput among users. In this paper, we propose, and analyze the performance of, three fairness enhancement schemes that can be integrated with link adaptation techniques. Results show that the three proposed scheme can enhance the fairness of link adaptation techniques with different degrees without causing (or with minor) degradation to the total network throughput.
\end{abstract}

Keywords-fairness; link adaptation techniques; adaptive power control; adaptive coding and modulation; radio resource management

\section{INTRODUCTION}

Unlike wireline links, wireless links experience significant temporal and spatial variation in the link quality. In wireless cellular networks, there are always disadvantaged users who are distant from their serving basestations or experiencing strong shadowing and/or deep fading. On the other hand, there are advantaged users who are close enough to their serving basestations and might be experiencing little/no shadowing and/or constructive fading. Furthermore, the disadvantaged users usually suffer from high interference levels since they are close enough to cochannel interferer basestations while the advantaged users usually have a low interference level since they are far away from cochannel interferer basestations. Wireless networks have conventionally been designed based on the worst case scenario such that all users including the disadvantaged ones are statistically guaranteed minimum quality of service in terms of the signal to interference ratio $(S I R)$. However, this approach is not efficient since it wastes part of the resources as the advantaged users often have much higher SIR than the minimum required value and these users do not make any use of it.

Link adaptation techniques have been proposed as a remedy of this signal quality variation. Adaptive power

This work has been supported by the National Capital Institute of Telecommunications (NCIT), Ottawa, Canada. control (PC) has been proposed to equalize the performance throughout the whole network by balancing the signal to interference ratio of all users (e.g. [1]). Alternatively, adaptive coding and modulation (ACM) has been proposed to exploit the variation in the signal quality by utilizing different coding and modulation levels to each user depending on SIR or any related parameter (e.g. [2]). Joint PC and ACM schemes have also been proposed for better resource utilization [3]. These link adaptation techniques vary in their fairness and efficiency performance. For instance, while PC tries to provide all users with almost the same signal quality and throughput, ACM yields largely varying signal quality and throughput. On the other hand, joint PC and ACM schemes can be considered as a balanced approach between the two extremes (PC and $\mathrm{ACM}$ ).

The performance of link adaptation techniques (PC, ACM, and joint $\mathrm{PC} \& \mathrm{ACM}$ ) are often analyzed in the literature focusing on the aggregate throughput as a metric of their efficiency without considering fairness among different users. Maximizing the aggregate throughput of adaptive modulation techniques subject to some fairness constraints has been considered in [4]. It has been shown that the problem is hard to be solved analytically. Therefore, the problem is heuristically divided into two sub-problems. The first part deals with the fairness by selecting a limited set of potential modulation levels for each user depending on the achieved time-averaged throughput. Hence, users having low timeaveraged throughput are compensated in the next frame by selecting higher modulation levels while those having high time-averaged throughput are assigned in the next frame lower modulation levels. The second part selects the modulation level of each user from the selected set given by part one with an objective of maximizing the total network throughput. However, results of achieved throughput or fairness performance have not been reported in [4].

In broadband fixed wireless networks, the fairness problem is more sever since users in unfavorable locations might stay there forever; hence the disadvantaged users might be always treated unfairly. On the other hand, long-term fairness in mobile networks is not a critical issue since a mobile user with a bad link at some instance will likely to have a good link subsequently (in the statistical sense). However, short-term fairness is not always attainable in mobile networks since 
some users might be stationary or having low mobility during their call duration.

In this paper, we propose three algorithms for enhancing the fairness of link adaptation techniques. Section II presents the proposed fairness enhancement techniques. Then, the results are provided in Section III. Section IV presents the system model. Finally the conclusions are given in Section V.

\section{PROPOSED TECHNIQUES}

Fairness enhancement can be achieved by either compensating the disadvantaged users, penalizing the advantaged users, or a combination of both. However, the fairness enhancement shouldn't come at the expense of efficiency degradation. Therefore, penalizing the advantaged users should be avoided (or at least minimized) to avoid reducing the total network throughput. The three proposed algorithms are simple and flexible enough to be easily integrated with any link adaptation technique with minimal implementation overhead. The proposed fairness enhancement techniques are as follows.

\section{A. Multiple Time Slots Allocation (MTSA)}

In this technique, fairness is enhanced by compensating users having instantaneous throughput less than a certain ratio of the ensemble-average throughput by an additional time slot. The ensemble-average throughput is a measure of the network performance at a specific time instant and it is given by

$$
M(j)=\frac{1}{N_{u}} \sum_{i=1}^{N_{u}} \operatorname{Thr}(i, j)
$$

where $\operatorname{Thr}(i, j)$ is the instantaneous throughput of user $i$ at frame $j$, and $N_{u}$ is the total number of users in the whole network. Then, the condition of assigning an additional time slot can be expressed as

$$
\operatorname{Thr}(i, j)<\alpha M(j)
$$

where $\alpha$ is a design parameter, where $(0<\alpha<1)$. At every frame, the resource management controller updates the ensemble-average throughput and then allocates additional time slots to disadvantaged users according to the condition given by (2). In addition to enhancing the fairness of link adaptation techniques, this technique can also improve the aggregate throughput as discussed in Section IV. However, it is apparent that this technique is less effective at high loading values since it becomes harder to find free additional time slots.

\section{B. Throughput Balancing (TB)}

This technique tries to balance the throughput in the same way that the binary $S I R$-balancing power control balances $S I R$ [5]. In this scheme, the resource management controller compares the instantaneous throughput of user $i$ at frame $j$ $(\operatorname{Thr}(i, j))$ with the ensemble-average throughput $(M(j))$. If
$\operatorname{Thr}(i, j)>M(j)$, the transmission rate in the next frame is reduced one step by moving the user to the next lower coding rate/modulation level combination as listed in Table I, otherwise it is increased one step. The incentive here is to keep the throughput of all users around the ensemble-average value. Hence, the allocated throughput is updated by

$$
\operatorname{Thr}(i, j+1)=\operatorname{Thr}(i, j)+\Delta \operatorname{Thr} \chi(\operatorname{Thr}(i, j)<M(j))
$$

where $\Delta T h r$ is the throughput step size, and $\chi(a<b)$ is an indicator function, which is equal to +1 if $a<b$ and -1 otherwise.

\section{Variable Maximum Power Constraint (VMPC)}

In this technique, throughput is equalized using the adaptation of the maximum power $\left(P_{\max }\right)$. Instead of having a fixed maximum power constraint for all users, the maximum power constraint in the power and rate selection is considered as a variable that depends on the relationship between user throughput $(\operatorname{Thr}(i, j))$ and ensemble-average throughput $(M(j))$. If $\operatorname{Thr}(i, j)<M(j)$, the maximum power of user $i$ at frame $j$ $\left(P_{\max }(i, j)\right)$ is increased by $\Delta P$, otherwise $P_{\max }(i, j)$ is reduced by $\Delta P$, where $\Delta P$ is a design parameter. Therefore, $P_{\max }$ is updated by

$$
P_{\text {max }}(i, j+1)=\min \left[P_{\text {abs_max }}, P_{\text {max }}(i, j)+\Delta P \chi\{\operatorname{Thr}(i, j)<M(j)\}\right]
$$

where $P_{a b s \text { max }}$ is the absolute (physical) maximum power. By adapting the maximum power, the disadvantaged users are compensated by increasing the maximum power that lead to throughput increase. Meanwhile, the advantaged users' maximum power is decreased, which yields throughput reduction.

\section{SYSTEM MODEL}

In order to quantify the fairness and efficiency of various link adaptation techniques, we define the fairness coefficient $(F C)$ as

$$
F C=\frac{1}{1+V}
$$

where $V$ is the variance of the throughput which is given by

$$
V=\frac{1}{N_{u}} \sum_{i=1}^{N_{u}}\left(\frac{1}{N_{f}} \sum_{j=1}^{N_{f}} \operatorname{Thr}(i, j)\right)^{2}-(E[M(j)])^{2}
$$

where $N_{f}$ is the total number of transmitted frames and $E[M(j)]$ is the time-average of the ensemble-average throughput $(M(j))$. It is apparent from this definition that the fairness 
coefficient $(F C)$ is inversely proportional to the throughput variance. Since the variance is a measure of the variation (unfairness) of the users' throughput, $F C$ is a valid measure of the fairness. Also, it should be noticed that $F C$ ranges from 0 (no fairness) to 1 (100\% fairness). The efficiency coefficient $(\eta)$ is defined as the ratio of time-average of the ensembleaverage of the throughput to the maximum throughput and it is given by

$$
\eta=\frac{E[M(j)]}{S_{\max }}
$$

where $S_{\max }$ is the maximum achievable throughput by a user.

As mentioned above the proposed techniques can be combined with any link adaptation technique. In this paper, the proposed fairness enhancement techniques are combined with joint power control (PC) and adaptive coding and modulation (ACM) algorithm using the selective power control policy (SPC) [6]. Eleven combinations of codingmodulation levels using Bit-Interleaved Coded Modulation (BICM) [7] are utilized to adapt the transmission rate to the channel conditions and interference variation. Table I lists these 11 coding-modulation combinations associated with their spectral efficiency and SIR requirements at $10^{-6}$ bit error rate (BER). The power level as well as the coding rate and modulation level are updated once per frame as follows

$$
P(i, j)=\max _{k}\left\{\frac{\delta P(i, j-1) \gamma^{k}}{\operatorname{SIR}(i, j-1)} \bar{\chi}\left(\frac{\delta P(i, j-1) \gamma^{k}}{\operatorname{SIR}(i, j-1)}<P_{\max }(i, j)\right)\right\}
$$

where $\gamma^{\xi}$ is the target SIR corresponding to the allocated coding rate and modulation level combination $(k), \delta$ is a constant used as a safety margin $(\delta>1)$, and $\bar{\chi}(a<b)$ is an indicator function which is equal to 1 if $a<b$, and 0 otherwise. This scheme tries to maximize the throughput by choosing the coding rate and modulation having the highest modulation efficiency based on the $S I R$ level of the previous frame. Meanwhile, the transmitted power is adjusted to achieve the corresponding SIR. Performance of SPC scheme without any fairness enhancement is also given in the results as a reference case.

\section{RESULTS}

Simulation is used to analyze the performance of the proposed techniques. A hexagonal cellular structure with 9 cells is considered. A wraparound grid is used to avoid the boundary effect. A TDMA system is assumed with 8 slots per frame. Directional antennas with $60^{\circ}$-beamwidth, $20 \mathrm{~dB}$ main lobe gain, and $10 \mathrm{~dB}$ side lobe gain are used at the basestation (BS). Similarly, subscriber stations (SSs) have directional antennas with $60^{\circ}$-beamwidth, $15 \mathrm{~dB}$ main lobe gain, and $0 \mathrm{~dB}$ side lobe gain. The channel model consists of exponential path loss model with an exponent $(n)$ of 4 , lognormal shadowing with a standard deviation $(\sigma)$ of $8 \mathrm{~dB}$, and temporallycorrelated flat Rayleigh fading samples generated using rounded (bell-shaped) Doppler spectrum with a 3-dB frequency of $2 \mathrm{~Hz}$ [8]. The Rayleigh fading samples of a user from different BSs are mutually independent. A frequency reuse plan of $1 / 6$ is employed such that the total spectrum is divided into 6 equal non-overlapping sub-bands allocated to the 6 sectors of each cell. The whole spectrum is reused in every cell. This relatively tight frequency reuse plan can be used because directional antennas are employed at both BSs and SSs.

Fig. 1 shows the efficiency coefficient $(\eta)$ of (SPC) with and without the three fairness enhancement techniques. It is apparent that MTSA enhances the efficiency coefficient $(\eta)$ considerably (by more than $20 \%$ ) especially at low to medium loading values. At high loading values, $\eta$ of MTSA approaches that of SPC without any fairness enhancement technique because of the unavailability of additional free time slots for compensating disadvantaged users. VMPC and TB schemes have almost the same efficiency coefficient $(\eta)$, which is less than that of SPC without fairness enhancement by $2-7 \%$ depending on the loading condition. Unlike MTSA case, both VMPC and TB cause slight reduction in the efficiency coefficient in order to enhance the fairness coefficient.

The fairness coefficient of the three proposed techniques (and SPC without any fairness enhancement technique) is depicted in Fig. 2. It is evident that the three proposed fairness enhancement techniques (MTSA, VMPC and TB) improve the fairness coefficient but with different ratios and different dependence on network loading. For instance, MTSA has the highest fairness coefficient $(F C)$ at low and medium loading values (less than 49\%). However, FC of MTSA is monotonically decreasing and approaching that of SPC due to the unavailability of additional free time slots at high loading. On the contrary, $F C$ of the other three cases (VMPC, TB, and no enhancement techniques) is decreasing with the increase of the network loading value until it reaches a minimum value at medium loading (between 30-40\%). However, by increasing the loading value, $F C$ starts to increase but with a slow pace. The reason for this trend is as follows. At low loading values, most users don't have high interference, and hence, they can achieve high throughput values, and this yields a high fairness coefficient $(F C)$. When the loading value increases, some users start to experience high interference and are enforced to use low throughput values, while others don't receive strong interference, and therefore, they still can have throughput values. This causes large variance, which yields low $F C$. At high loading values, most of the users have high interference and as a result, they achieve close values of low throughput. This similarity reduces the variance again, which yields higher $F C$. $F C$ of MTSA does not have such a minimum at medium loading values because MTSA can use free slots at medium loading values to compensate the disadvantaged users. It is also worth mentioning that at medium to high loading values, VMPC has the highest $F C$, which is higher than that of SPC without enhancement techniques by more than $60 \%$ at $100 \%$ loading. 


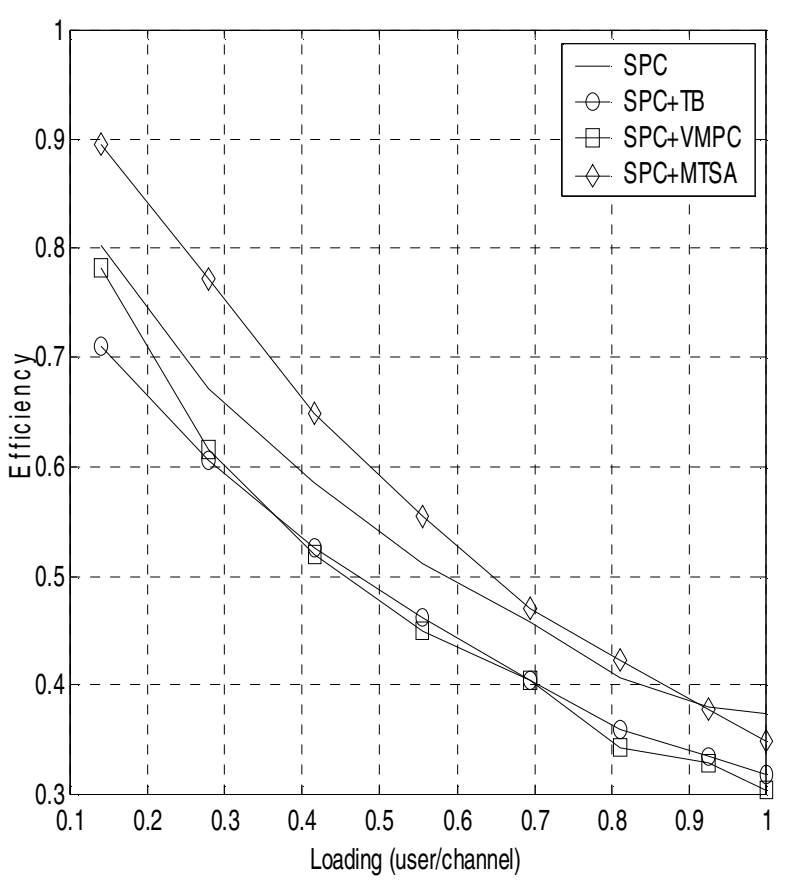

Fig.1. Efficiency of joint PC \& ACM scheme (SPC) with and without fairness enhancement.

TABLE I. SIR OF DIFFERENT CODING- MODULATION LEVELS

\begin{tabular}{|c|c|c|c|}
\hline $\begin{array}{c}\text { Coding rate } \\
\text { \& modulation } \\
\text { level index } \\
(\boldsymbol{k})\end{array}$ & $\begin{array}{c}\text { Coding rate \& } \\
\text { Modulation } \\
\text { level } \\
\text { combination }\end{array}$ & $\begin{array}{c}\text { Spectral } \\
\text { Efficiency } \\
(\mathbf{b} / \mathbf{s} / \mathbf{H z})\end{array}$ & $\begin{array}{c}\text { SIR at 10 } \\
\text { BER } \\
\left(\boldsymbol{\gamma}^{\boldsymbol{k}}\right) \mathbf{~ d B}\end{array}$ \\
\hline 1 & $1 / 2$ \& QPSK & 1.00 & 4.65 \\
\hline 2 & $2 / 3$ \& QPSK & 1.33 & 6.49 \\
\hline 3 & $3 / 4$ \& QPSK & 1.50 & 7.45 \\
\hline 4 & $7 / 8$ \& QPSK & 1.75 & 9.05 \\
\hline 5 & $1 / 2 \& 16-\mathrm{QAM}$ & 2.00 & 10.93 \\
\hline 6 & $2 / 3 \&$ 16-QAM & 2.66 & 12.71 \\
\hline 7 & $3 / 4 \&$ 16-QAM & 3.00 & 14.02 \\
\hline 8 & $7 / 8 \&$ 16-QAM & 3.50 & 15.74 \\
\hline 9 & $2 / 3 \& 64-\mathrm{QAM}$ & 4.00 & 18.50 \\
\hline 10 & $3 / 4 \& 64-\mathrm{QAM}$ & 4.50 & 19.88 \\
\hline 11 & $7 / 8 \& 64-\mathrm{QAM}$ & 5.25 & 21.94 \\
\hline
\end{tabular}

\section{CONCLUSIONS}

Three fairness enhancement techniques have been proposed and analyzed in this paper. It has been shown that the fairness of link adaptation techniques can be enhanced without degrading the efficiency as in the case of MTSA or with a little reduction in the efficiency as in the case of VMPC and TB. At low loading values, MTSA is the most effective in enhancing the fairness, while at medium to high loading values VMPC is the best in terms of the fairness coefficient. A combined scheme of MTSA and VMPC will be considered for future work aiming to achieve high fairness at different

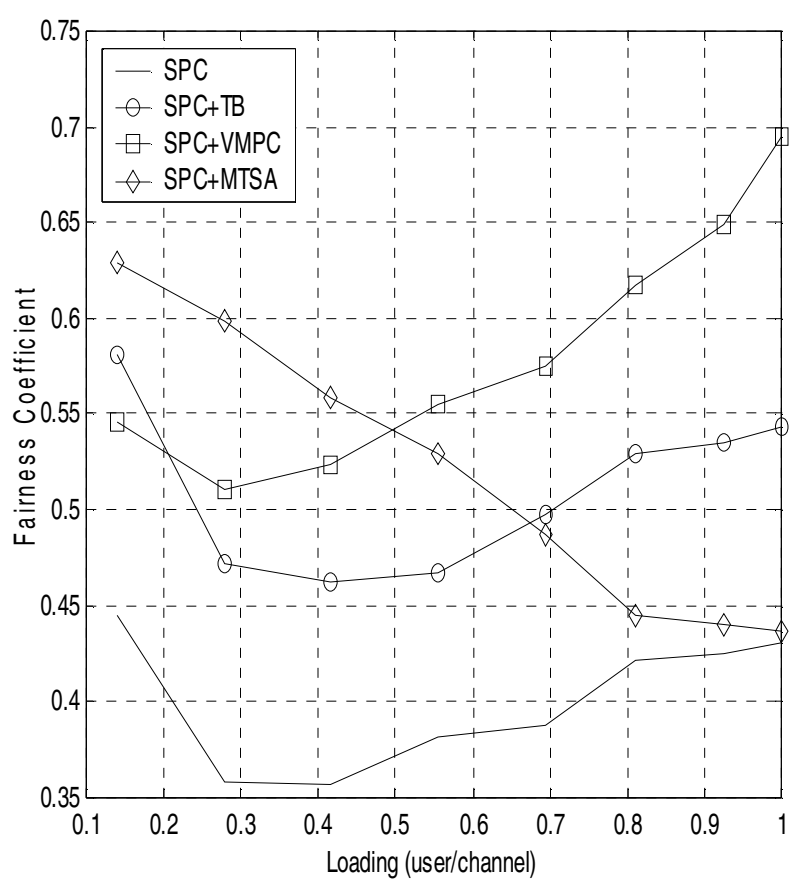

Fig.2. Fairness coefficient of Joint PC \& ACM scheme (SPC) with and without Fairness enhancement.

loading values. The use of double threshold TB scheme will be also investigated.

\section{ACKNOWLEDGEMENT}

We would like to thank Dr. Sirikiat Lek Ariyavisitakul for providing us with the $S I R$ values of different coding rate and modulation level combinations listed in Table I.

\section{REFERENCES}

[1] J. Zander, "Performance of optimum transmitter power control in cellular radio systems," Proc. IEEE Trans. on Vehicular Technology, vol. 41, no. 1, pp. 57-62, Feb. 1992.

[2] A. Goldsmith and S. Chua, "Adaptive coded modulation for fading channels," IEEE Trans. on Commun., vol. 46, no. 5, pp. 595-602, May 1998.

[3] S. Kim, Z. Roseberg, and J. Zander, "Combined power control and transmission rate selection in cellular networks," Proc. IEEE Vehicular Technology Conference Fall (VTC), pp. 1653-1657, Sept. 1999.

[4] Z. Han and R. Liu, "Throughput maximization using adaptive modulation in wireless networks with fairness constraints," Proc. IEEE Wireless Communications and Networking Conference (WCNC), pp. 243-246, March 2003.

[5] S. Ariyavisitakul, "SIR-based power control in a CDMA system," Proc. IEEE Globecom'92, pp. 868-873, Dec.1992.

[6] R. Jantti and S. Kim, "Selective power control with active link protection for combined rate and power management," Proc. IEEE Vehicular Technology Conference Spring (VTC), pp. 1960-1964, May 2000.

[7] G. Cair, G. Taricco, and E. Biglieri, "Bit-interleaved coded modulation," Proc. IEEE Trans. on Info. Theory, vol. 44, no. 3, pp. 927-946, May 1998.

[8] D. Baum, "Simulating the SUI channel models," IEEE 802.16.3c-01/53, April 2001. 